\title{
Prévention de la violence et promotion des relations harmonieuses et égalitaires : regard croisé des membres du personnel et des étudiantes et étudiants de niveau collégial
}

\author{
Mylène Fernet et Martine Hébert \\ Université du Québec à Montréal
}

\author{
Jean-François Cardinal, Huguette Guay, Danielle Bédard et Geneviève Perreault \\ Cégep Marie-Victorin
}

\begin{abstract}
RÉSUMÉ
La violence dans les relations amoureuses (VRA) est associée à d'importantes répercussions sur la santé mentale des jeunes. Considérant le peu d'interventions préventives sur la VRA leur étant destinées, cette étude vise à contraster les perspectives du personnel collégial $(n=57)$ et des étudiants et étudiantes $(n=278)$. Les résultats suggèrent des connaissances plutôt justes par rapport à la violence et des attitudes positives face à la prévention. Toutefois, certaines barrières sont identifiées, dont le manque d'outils de prévention et la surcharge de travail. Ces résultats renforcent la nécessité d'offrir des activités de formation et des outils pédagogiques pour soutenir la prévention de la VRA en milieu collégial.
\end{abstract}

Mots clés : violence dans les relations amoureuses, jeunes adultes, prévention, promotion de la santé

Mylène Fernet, Ph.D., Département de sexologie, Université du Québec à Montréal; Martine Hébert, Ph.D., Département de sexologie, Université du Québec à Montréal; Jean-François Cardinal, Mps., Département de travail social, Cégep Marie-Victorin; Huguette Guay, t.s., Département de travail social, Cégep Marie-Victorin; Danielle Bédard, MÉ.D., M.A., CSSS Pointe-de-L'île, Service des affaires étudiantes, Cégep Marie-Victorin; Geneviève Perreault, M.A., Département sciences sociales, Cégep Marie-Victorin.

Cette recherche découle d'un soutien financier du Centre de recherche interdisciplinaire sur la violence familiale et la violence faite aux femmes (CRI-VIFF) et de l'Équipe ÉVISSA (Équipe sur la violence sexuelle et la santé). Les auteurs souhaitent remercier les étudiantes et étudiants ainsi que les membres du personnel qui ont si généreusement accepté de participer à cette étude. Ils tiennent aussi à remercier mesdames Odette Lussier et Catherine Paquin-Boivin pour leur participation au processus de recherche, de même qu'à souligner la précieuse collaboration des professionnelles de recherche, Manon Robichaud et Mélanie St-Hilaire, lors des étapes de rédaction.

La correspondance concernant cet article devrait être acheminée à Mylène Fernet, Département de sexologie, Université du Québec à Montréal, Montréal (Québec), Canada, Tél.: (514) 987-3000\#2201, Fax: (514) 987-6787, courriel: fernet.mylene@uqam.ca 


\begin{abstract}
Dating violence (DV) is associated with significant negative impacts on young adults' mental health. There are few initiatives for preventing DV targeting youth in colleges. This study's objective is to contrast the perspectives of college personnel $(n=57)$ with those of students $(n=278)$ regarding DV prevention. Results suggest that participants have a relatively high level of knowledge regarding violence and a positive attitude toward the benefits of prevention endeavours. However, school personnel feel that they don't have enough prevention tools and are overworked. These results highlight the need to offer educational activities to support DV prevention efforts in colleges.
\end{abstract}

Keywords: dating violence, young adults, prevention, health promotion

La violence dans les relations amoureuses (VRA) est un problème important chez les jeunes. Elle réfère à toute agression physique, sexuelle ou psychologique commise délibérément par un individu à l'endroit de la personne qu'il fréquente. Il peut s'agir tout aussi bien de relations occasionnelles que de relations de longue date (Centre national d'information sur la violence dans la famille du Canada, 2006). Au Québec, aucune donnée représentative sur la prévalence de la violence dans les relations amoureuses n'est actuellement disponible pour les populations de jeunes adultes. Pourtant, selon le Ministère de la Sécurité publique du Québec (2009), 22 \% des plaintes déposées à la police pour violence conjugale et jugées fondées après enquête en 2008 concernaient des jeunes, majoritairement des femmes âgées de 18 à 24 ans.

Chez les plus jeunes, les données représentatives obtenues à partir des témoignages des adolescents et adolescentes indiquent que chez les filles de 16 ans qui ont fréquenté un partenaire au cours des 12 mois précédant l'étude, environ une fille sur trois (34\%) rapporte avoir vécu de la violence psychologique, une sur cinq (20\%) de la violence physique et une sur dix (11\%) de la violence sexuelle (Lavoie et Vézina, 2002). Une enquête représentative des jeunes de secondaire I, III et V des écoles de l'île de Montréal révèle qu'un quart ( $27 \%$ ) des élèves de secondaire I et plus d'un tiers ( $40 \%$ ) des élèves de secondaire III et V rapportent avoir vécu des expériences de violence, qu'il s'agisse de violence subie ou infligée (Riberdy et Tourigny, 2009).

Au Québec, les interventions de prévention de la VRA et de promotion des relations amoureuses harmonieuses et égalitaires (RAHE) sont peu nombreuses en milieu collégial. En fait, les interventions ou programmes de prévention de la VRA sont généralement offerts aux plus jeunes dans les écoles secondaires ou par les organismes communautaires travaillant avec ces mêmes jeunes (Lavoie, 2000). Une recension récente et exhaustive des programmes de prévention de la VRA offerts aux élèves de niveau secondaire (Lavoie, Hébert et Beaulieu-Denault, 2012) identifie les programmes de courte durée comprenant entre deux et cinq rencontres, d'après un contenu préétabli et destiné à un auditoire mixte dans des classes obligatoires, comme étant la formule la plus couramment privilégiée tant au Québec qu'aux États-Unis. Ces programmes visent généralement à amener les participants et participantes à revoir leurs attitudes et leurs connaissances et à identifier des signes annonciateurs de la violence dans leurs relations amoureuses et des ressources potentielles (Avery-Leaf, Cascardi, O’Leary et Cano, 1997; Lavoie, Hotton-Paquet, Laprise et Joyal Lacerte, 2009). D'autres programmes ciblent à la fois les jeunes qui pourraient faire face à une situation de violence et ceux et celles qui pourraient potentiellement être témoins de violence et appelés à agir comme confidents et confidentes (Lavoie, Pacaud, Roy et Lebossé, 2007). 
Les interventions de prévention de la VRA et de promotion des RAHE offertes spécifiquement aux jeunes de niveau collégial sont beaucoup plus rares. Par exemple, aux États-Unis, les travaux de Banyard, Plante et Moynihan (2004) ont souligné l'importance d'élaborer des programmes s'adressant à d'éventuels témoins de situations de violence afin de les outiller à jouer un rôle actif de dénonciation et de soutien des victimes. Ces auteurs postulent qu'il est possible d'avoir un impact positif sur les attitudes face à la violence et sur la façon dont les jeunes adultes se représentent les normes sociales et les attentes de leurs pairs en promouvant les habilités positives associées au rôle de pair aidant, c'est-à-dire reconnaître la violence et savoir intervenir sans compromettre sa propre sécurité.

À notre connaissance, au Québec, aucun programme défini au sens « d'une combinaison coordonnée de plusieurs interventions complémentaires, élaborées afin de renforcer un message visant divers milieux » (Lavoie, 2000, p. 424) ne cible spécifiquement les jeunes de niveau collégial. Par contre, quelques outils de sensibilisation sont disponibles, par exemple, Épris . . sans mépris. Des campagnes de sensibilisation telles L'amour donne des ailes, il ne les coupe pas et L'amour en douceur : c'est l'affaire de chacun des partenaires ont été réalisées et ont donné lieu à des affiches et dépliants à l'intention des jeunes. Plusieurs activités d'animation se tiennent ponctuellement dans les établissements d'enseignement collégial à travers le Québec, par exemple, des activités photo ou des kiosques à la Saint-Valentin abordant les relations amoureuses mais sans viser spécifiquement la prévention de la VRA. Ce survol des programmes destinés aux jeunes de niveau collégial n'a pas la prétention d'être exhaustif mais permet toutefois de constater que les effets de ces activités de prévention de la VRA ne sont généralement pas évalués. La plupart d'entre eux sont de nature ponctuelle et abordent la question de la VRA de façon indirecte. Pourtant, l'intensité de l'exposition, qui doit être suffisante pour permettre d'en apprécier les effets, est reconnue comme étant un critère d'efficacité des activités de prévention destinées aux jeunes (Nation et al., 2003). En effet, il apparaît que les programmes offrant de multiples sessions et des interventions de plus longue durée sont plus efficaces (Ting, 2009).

Bowen et Desbiens (2004) soulignent que les pratiques de prévention de la violence en milieu scolaire primaire et secondaire reposent sur une juste connaissance de l'ampleur du phénomène et de ses manifestations dans différents contextes. Et au-delà des facteurs d'ordre individuel, socio-économique ou socioculturel, l'école contribue de façon significative à l'expression et à la réduction du problème entre ses murs. Par conséquent, il devient essentiel d'identifier les pratiques et les attitudes des adultes qui contribuent à prévenir ou à accroître l'expression des conduites violentes. Dans l'optique d'orienter le développement d'interventions préventives de la VRA et de promotion des RAHE chez les jeunes et d'optimiser les conditions d'implantation des programmes en milieu collégial, il nous apparaissait nécessaire d'obtenir un regard croisé sur les perceptions respectives de deux groupes d'acteurs directement interpellés par la question. La présente étude vise à : (a) contraster les connaissances des membres du personnel avec celles des étudiantes et étudiants à propos de la VRA; (b) contraster les attitudes de ces groupes à l'égard de la promotion des RAHE; (c) identifier les barrières à aborder la VRA rencontrées par les membres du personnel; (d) identifier les intérêts des étudiantes et étudiants par rapport aux moyens de promotion des RAHE; (e) décrire les besoins d'information et d'intervention des étudiantes et étudiants en lien avec la thématique des RAHE; et (f) décrire les intérêts des membres du personnel à l'égard de la prévention de la VRA. Ces informations sont susceptibles d'offrir des pistes pertinentes pour l'implantation d'initiatives de prévention en milieu collégial. 


\section{MÉTHODOLOGIE}

\section{Participantes et participants}

Les données ont été recueillies auprès de la communauté étudiante et du personnel d'un établissement d'enseignement collégial de la région métropolitaine de Montréal. Tous les participantes et participants sont volontaires et ont donné un consentement libre et éclairé.

Membres du personnel. L'échantillon des membres du personnel $(n=57)$ est constitué d'enseignantes et d'enseignants (70,2\%), de professionnelles et professionnels (15,8\%), de cadres $(5,3 \%)$ et d'employées et d'employés de soutien (7\%), tous interpellés par la VRA. Une stagiaire $(1,7 \%)$ a également répondu au questionnaire. L'échantillon est constitué de 14 hommes (24,6\%) et 43 femmes (75,4\%). Dans ceux-ci et celles-ci, 1,8\% étaient âgés entre 20 et 29 ans, $15,8 \%$ entre 30 et 39 ans, $45,6 \%$ entre 40 et 49 ans, puis 36,8 \% entre 50 et 59 ans. La majorité a complété des études universitaires (baccalauréat 45,6\%, maîtrise 42,1\%, doctorat 3,5\%). Parmi les enseignantes et enseignants interrogés, plus de la moitié $(54,8 \%)$ proviennent du programme des techniques humaines.

Étudiantes et étudiants. Au total, 284 étudiantes et étudiants ont rempli le questionnaire. Puisque la présente étude porte sur les relations amoureuses harmonieuses et égalitaires des étudiantes et étudiants de niveau collégial (versus les relations conjugales), les répondantes et répondants âgés de 31 ans et plus ont été retirés de l'échantillon $(n=6)$. L'échantillon final est donc constitué de 278 étudiantes et étudiants, soit $84,4 \%$ femmes et 15,6\% hommes. La majorité est âgés de 20 ans et moins (18 ans et moins, 43,6\%; 19-20 ans, 41,8\%; 21-25 ans, 10,2\%; 26-30 ans, 4,4\%). Quant à leur scolarité, 65,1\% ont complété les études secondaires et $34,5 \%$ un programme collégial; $0,4 \%$ ont terminé un programme universitaire. Concernant leurs études collégiales actuelles, 42,5\% sont dans un programme d'études du secteur préuniversitaire et $57,5 \%$ dans un programme du secteur technique. Un peu plus de la moitié sont en relation amoureuse au moment de l'étude $(57,7 \%)$, et cela, en moyenne, depuis 20,13 mois $(E ́ T=18,08)$. La durée des relations rapportées varie d'une semaine à 8 ans.

\section{Mesures}

Une concertation de l'équipe de recherche provenant des milieux universitaire et collégial a permis de cerner les indicateurs pertinents. Un questionnaire auto-administré distinct a été conçu pour chacun des groupes pour les besoins de l'étude, mais certains instruments de mesure sont communs aux deux groupes.

Connaissances concernant la VRA. Seize items permettent d'évaluer les connaissances des participantes et participants (membres du personnel et étudiantes et étudiants) par rapport à la VRA. Ces items incluent : " Dans une relation amoureuse, une personne qui violente son partenaire tente de le contrôler »; «Si une jeune femme subit de la violence dans le contexte d'une relation amoureuse, c'est parce qu'elle a fait une action pour provoquer cette situation »; «La violence dans les relations amoureuses des jeunes adultes n'est pas associée à des conséquences aussi sévères que la violence dans les relations conjugales d'adultes plus âgés ». L'ensemble des items administrés ont été adaptés d'instruments de mesure existants : 11 items provenant de l'inventaire utilisé dans le programme Skills for Violence-Free Relationships (Krajewski, Rybarik, Dosch et Gilmore, 1996), 3 items du questionnaire sur les connaissances au sujet de l'agression 
sexuelle (Laforest, 2001), un item du questionnaire utilisé dans le programme VIRAJ (Lavoie, Vézina, Gosselin et Robitaille, 1994) et un item élaboré spécifiquement pour la présente étude.

Attitudes à l'égard de la promotion des RAHE. Un ensemble de 11 items ont été utilisés pour évaluer les attitudes sur la promotion des RAHE. Ces items incluent : " Une initiative de prévention dispensée en milieu collégial pourrait aider à prévenir la violence dans les relations amoureuses chez les étudiants »; «Une initiative de prévention soutenue par le personnel enseignant peut être aussi efficace que si elle est offerte par un ou une spécialiste dans le domaine »; "Ce sont uniquement aux filles que doivent être adressées les initiatives de prévention de la violence dans les relations amoureuses ». Ces items ont été inspirés des travaux de St-Vincent (2002). Par ailleurs, des trois items s'adressant spécifiquement aux étudiantes et étudiants ont été ajoutés dans le but d'évaluer leurs perceptions du rôle potentiel des amies et amis et des parents dans la promotion des RAHE chez les cégépiennes et cégépiens. Ces items ont été spécifiquement élaborés pour la présente étude.

Barrières à aborder la VRA. Treize items évaluent les barrières à aborder la VRA telles que perçues par les membres du personnel. Ces items incluent : «Trop peu d'étudiants vivent des situations de violence dans les relations amoureuses pour en faire un thème de discussion »; " J'aborderais le sujet seulement si j'avais des doutes qu'un étudiant soit victime de violence dans sa relation amoureuse »; « Je considère une initiative de prévention faisant appel aux pairs comme étant une option à privilégier en milieu collégial ». Neuf items proviennent d'une adaptation des travaux de St-Vincent (2002); trois proviennent de ceux de Hamelin-Brabant, Damant, Michaud et Alain (2007); et un item a été élaboré pour la présente étude.

Les items de ces trois instruments (connaissances concernant la VRA, attitudes à l'égard de la promotion des RAHE et barrières à aborder la VRA) sont complétés à l'aide d'une échelle de réponse de type Likert à 4 niveaux variant de 1 (Fortement en accord) à 4 (Fortement en désaccord). Pour le besoin des analyses, les données ont été transformées afin d'obtenir une échelle de réponse dichotomique, soit en accord/vrai pour 1 et 2 (Fortement en accord et Légèrement en accord) vs en désaccord/faux pour 3 et 4 (Légèrement en désaccord et Fortement en désaccord).

Intérêt par rapport aux moyens de promotion des RAHE. Inspirés des travaux de St-Vincent (2002), nous avons interrogé les étudiantes et étudiants sur leur intérêt à l'égard des moyens d'intervention dans un contexte collégial pour la promotion des RAHE. Quinze items se répartissent en trois catégories, soit des outils d'information (par exemple, brochures distribuées aux étudiantes et étudiants sur les relations amoureuses saines), des animations hors cours ou en classe (par exemple, proposition de sites Internet sur les relations amoureuses saines) et un service d'aide et de consultation (par exemple, «présence d'un intervenant de milieu qui peut nous écouter ou nous conseiller, entre autres quant aux questions touchant aux relations amoureuses »). Le choix de réponse est dichotomique (oui/non).

Besoins liés à la thématique des RAHE. Les étudiantes et étudiants ont complété une section concernant leurs besoins d'information et leurs besoins d'intervention. Les travaux de St-Vincent (2002) ont inspiré l'élaboration d'une liste de 18 thématiques pouvant être abordées, reliées aux relations amoureuses (voir le tableau 5 pour les thèmes proposés).

Intérêts face à la prévention de la VRA. Cette échelle sur les intérêts des membres du personnel comporte six items et est une adaptation des travaux de St-Vincent (2002). Chacun des thèmes suggérés aux 
membres du personnel est présenté au tableau 6. Un item supplémentaire abordant spécifiquement l'intérêt des membres du personnel à suivre une formation sur la VRA a été ajouté à cette section.

Pour ces deux derniers instruments (besoins liés à la thématique des RAHE et intérêts face à la prévention de la VRA), l'échelle de réponse est de type Likert en quatre points variant de 1 (Pas intéressé) à 4 (Très intéressé). À partir de cette échelle, les données ont été regroupées selon les catégories « Pas et peu intéressé » et «Intéressé et très intéressé ». Seul le dernier item de l'instrument de mesure sur les intérêts des membres du personnel à suivre une formation sur la VRA a un choix de réponse dichotomique (oui/non).

\section{Procédures}

Le recrutement des membres du personnel a été effectué par les collaboratrices et collaborateurs de l'établissement collégial, par contact téléphonique, en personne ou par courriel, les conviant à une rencontre pédagogique d'une demi-journée sous le thème de la sensibilisation à la thématique des RAHE. Lors de cette rencontre, les membres du personnel enseignant, professionnel et de soutien ainsi que le personnel cadre ont été invités à participer à l'étude. Les membres du personnel intéressés ont été ensuite invités à participer à un groupe de discussion sur le thème de la prévention de la VRA et de la promotion des RAHE et devaient, au préalable, remplir un questionnaire auto-administré qui nécessitait environ 15 minutes.

Le recrutement des étudiantes et étudiants a été effectué par les collaboratrices et collaborateurs du milieu dans le cadre du cours d'éducation physique. L'invitation a été lancée pour chaque groupe-classe (14 groupes). Cette stratégie de recrutement a favorisé la rencontre des étudiantes et étudiants de tous les niveaux d'étude et à différentes étapes du cheminement scolaire. La complétion du questionnaire exigeait environ 20 minutes.

Les mineurs (moins de 18 ans) intéressés à participer devaient, au préalable, obtenir le consentement d'un parent ou tuteur pour participer à la collecte de données qui avaient lieu la semaine suivante. Tous les participants et participantes ont, au préalable, signé un formulaire de consentement. Le présent projet a reçu l'approbation du comité institutionnel de la recherche (CIER) de l'Université du Québec à Montréal et de celui de l'établissement collégial participant. Aucune compensation financière n'a été allouée. Les étudiantes et étudiants ont pu bénéficier d'un résumé des résultats, sous forme de capsules pédagogiques.

\section{RÉSULTATS}

\section{Connaissances concernant la VRA}

Les pourcentages de bonnes réponses sur l'échelle de connaissances concernant la VRA sont présentés au tableau 1. Des analyses de khi-carré ont été effectuées sur les items communs afin de comparer le niveau de connaissances des étudiantes et étudiantes avec celui des membres du personnel. Les résultats révèlent que les étudiantes et étudiants et membres du personnel ont, en général, des connaissances justes par rapport au phénomène de la VRA. Ainsi, la majorité des participantes et participants indiquent qu'il est aussi possible dans le contexte d'une relation amoureuse d'être victime de violence psychologique que de violence physique, qu'il est faux de penser que la victime a provoqué la violence par une action et que la VRA peut être présente malgré l'absence d'actes témoignant de violence physique. Par ailleurs, la très grande majorité (plus de $90 \%$ ) croit que les victimes de violence craignent généralement de dévoiler les 


\section{Tableau 1}

\section{Répartition des pourcentages de bonnes réponses à l'échelle de connaissances sur la violence} dans les relations amoureuses et comparaison des deux groupes sur les items communs

\begin{tabular}{|c|c|c|c|c|c|}
\hline & \multicolumn{2}{|c|}{ Personnel } & \multicolumn{2}{|c|}{ Étudiant(e)s } & \multirow{2}{*}{$\begin{array}{l}\text { Test statistique } \\
\chi_{(1)}^{2}\end{array}$} \\
\hline & $N$ & $\%$ & $N$ & $\%$ & \\
\hline $\begin{array}{l}\text { Dans une relation amoureuse, il est aussi possible d'être victime } \\
\text { de violence psychologique que de violence physique }(V)\end{array}$ & 57 & 100,0 & 277 & 99,3 & 0,414 \\
\hline $\begin{array}{l}\text { Si une jeune femme subit de la violence dans le contexte d'une } \\
\text { relation amoureuse, c'est parce qu'elle a fait une action pour } \\
\text { provoquer cette situation }(\mathrm{F})\end{array}$ & 57 & 100,0 & 277 & 97,1 & 1,687 \\
\hline $\begin{array}{l}\text { Dans une relation amoureuse, tant qu'il n'y a pas de violence } \\
\text { physique, il n'y a pas vraiment de violence (F) }\end{array}$ & 57 & 100,0 & 277 & 96,8 & 1,903 \\
\hline $\begin{array}{l}\text { Les victimes de violence dans leurs relations amoureuses } \\
\text { craignent généralement de dévoiler l'incident }(\mathrm{V})\end{array}$ & 57 & 93,0 & 277 & 96,8 & 1,795 \\
\hline $\begin{array}{l}\text { La plupart des cas de violence dans les relations amoureuses } \\
\text { sont rapportés aux autorités policières (F) }\end{array}$ & 57 & 100,0 & 275 & 92,0 & $4,884^{*}$ \\
\hline $\begin{array}{l}\text { Il est facile pour la victime de quitter une relation amoureuse } \\
\text { violente lorsqu'elle le décide }(\mathrm{F})\end{array}$ & 54 & 94,4 & 277 & 91,0 & 0,703 \\
\hline $\begin{array}{l}\text { Au fil du temps, le cycle de violence dans les relations } \\
\text { amoureuses tend vers une intensification }(\mathrm{V})\end{array}$ & 57 & 93,0 & 275 & 90,5 & 0,341 \\
\hline $\begin{array}{l}\text { Dans une relation amoureuse, une personne qui violente son } \\
\text { partenaire tente de le contrôler }(\mathrm{V})\end{array}$ & 57 & 96,5 & 275 & 89,1 & 2,969 \\
\hline $\begin{array}{l}\text { Lorsqu'une personne se sent effrayée, menacée ou contrôlée par } \\
\text { les propos ou les actions d'une autre personne, il s'agit d'une } \\
\text { relation amoureuse violente }(\mathrm{V})\end{array}$ & 57 & 94,7 & 278 & 88,5 & 1,973 \\
\hline $\begin{array}{l}\text { Dans une relation amoureuse, une personne qui se fait souvent } \\
\text { insulter par son ou sa partenaire est victime de violence (V) }\end{array}$ & 57 & 94,7 & 276 & 88,0 & 2,195 \\
\hline $\begin{array}{l}\text { Les victimes de violence dans leurs relations amoureuses pro- } \\
\text { viennent autant de milieux socio-économiques favorisés que de } \\
\text { défavorisés (V) }\end{array}$ & 57 & 93,0 & 277 & 86,3 & 1,931 \\
\hline $\begin{array}{l}\text { La violence dans les relations amoureuses des jeunes adultes } \\
\text { n'est pas associée à des conséquences aussi sévères que la } \\
\text { violence dans les relations conjugales d'adultes plus âgés (F) }\end{array}$ & 57 & 84,2 & 277 & 86,3 & 0,168 \\
\hline $\begin{array}{l}\text { Les victimes de violence dans leurs relations amoureuses } \\
\text { le dévoilent habituellement à quelqu'un peu de temps après } \\
\text { l'incident (F) }\end{array}$ & 57 & 89,5 & 271 & 84,1 & 1,057 \\
\hline $\begin{array}{l}\text { Les jeunes femmes sont plus à risque d'être victimes de violence } \\
\text { dans leurs relations amoureuses que les jeunes hommes }(\mathrm{V})\end{array}$ & 57 & 94,7 & 275 & 78,9 & $7,887^{* *}$ \\
\hline $\begin{array}{l}\text { Les victimes de violence dans leurs relations amoureuses sont } \\
\text { souvent moins scolarisées }(\mathrm{F})\end{array}$ & 57 & 64,9 & 277 & 83,8 & $10,708^{* * *}$ \\
\hline $\begin{array}{l}\text { La violence a plus de probabilités d'apparaître dans une relation } \\
\text { lorsqu'un des partenaires a plus de pouvoir et de contrôle que } \\
\text { l'autre (V) }\end{array}$ & 56 & 85,7 & 277 & 75,1 & 2,958 \\
\hline
\end{tabular}

$* \leq 0,05 ; * * \leq 0,01 ; * * * \leq 0,001$ 
actes vécus et que la violence tend à s'intensifier avec le temps. La majorité des membres du personnel et des étudiantes et étudiants reconnaissent par ailleurs qu'il n'est pas facile pour une personne qui subit de la VRA de mettre fin et quitter la relation.

L'analyse des données révèle, par contre, que certaines notions liées à la VRA semblent moins bien maîtrisées ou connues ou que les participantes et participants affichent encore certains mythes. Ainsi, une proportion non négligeable (c.-à-d., environ $15 \%$ et plus) du groupe étudiant et celui des membres du personnel croient, à tort, que le rapport de pouvoir et de contrôle entre les partenaires a peu ou pas d'effet sur l'apparition de la violence, que les victimes dévoilent habituellement à quelqu'un peu de temps après l'incident et que les conséquences de la VRA ne sont pas aussi sévères que celles de la violence conjugale.

Toutefois, certaines différences sont identifiées entre les perceptions du groupe étudiant et celui du personnel. En effet, les membres du personnel semblent plus portés à croire que les victimes de la VRA sont moins scolarisées. Bien que le taux de bonnes réponses soit élevé pour les deux groupes de répondantes et répondants, les étudiantes et étudiants sont plus susceptibles de croire, à tort, que la plupart des cas de VRA sont rapportés aux autorités policières. Finalement, alors que la vaste majorité $(94,7 \%)$ des membres du personnel scolaire considèrent que les jeunes femmes sont plus à risque d'être victimes de VRA que les jeunes hommes, $78,9 \%$ des étudiantes et étudiants sont de cet avis.

\section{Attitudes à l'égard de la prévention de la VRA}

Le tableau 2 présente les pourcentages de membres du personnel et d'étudiantes et étudiants présentant des attitudes positives à l'égard de la prévention de la VRA. En général, les deux groupes entretiennent des attitudes positives quant aux bénéfices potentiels des initiatives de prévention. Ainsi, la vaste majorité (plus de $95 \%$ ) considère que les initiatives de prévention ont leur place au cégep, qu'elles doivent s'adresser autant aux jeunes femmes qu'aux jeunes hommes, qu'elles pourraient les aider à reconnaître des situations de violence et que tous les cégeps devraient proposer et offrir des initiatives de prévention de la VRA.

Par contre, un pourcentage non négligeable considère que les initiatives de prévention peuvent être associées à certains effets négatifs chez les étudiantes et étudiants. En ce sens, près du quart des membres du personnel ainsi que des étudiantes et étudiants considèrent que la participation à une initiative de prévention pourrait faire en sorte que les étudiantes et étudiants soient plus réticents à développer une relation intime avec un ou une partenaire.

Par ailleurs, les données indiquent certaines distinctions quant aux attitudes des deux groupes face à d'éventuelles initiatives de prévention. Ces différences réfèrent aux énoncés évaluant l'intérêt des étudiantes et étudiantes, le rôle des enseignantes et enseignants et les effets des initiatives de promotion auprès des étudiantes et étudiants. Ainsi, les membres du personnel sont moins nombreux à croire que les initiatives de prévention suscitent fortement l'intérêt des jeunes que les étudiantes et étudiants eux-mêmes. Par ailleurs, alors que tous les membres du personnel scolaire croient qu'une initiative de prévention pourrait aider à prévenir la VRA et motiver les jeunes à dévoiler une situation de violence qu'ils vivent, un pourcentage moins élevé des étudiantes et étudiants sont de cet avis.

Une proportion plus importante des étudiantes et étudiants que des membres du personnel scolaire considèrent qu'une initiative de prévention peut mener les jeunes à développer une vision négative des relations 


\section{Répartition des pourcentages des attitudes favorables à l'égard de la prévention de la violence dans les relations amoureuses et comparaison des deux groupes sur les items communs}

\begin{tabular}{|c|c|c|c|c|c|}
\hline & \multicolumn{2}{|c|}{ Personnel } & \multicolumn{2}{|c|}{ Étudiant(e)s } & \multirow{2}{*}{$\begin{array}{l}\text { Test statistique } \\
\chi_{(1)}^{2}\end{array}$} \\
\hline & $N$ & $\%$ & $N$ & $\%$ & \\
\hline $\begin{array}{l}\text { Les initiatives de prévention n'ont pas leur place au cégep } \\
\text { (en désaccord) }\end{array}$ & 57 & 98,2 & 277 & 98,2 & 0,001 \\
\hline $\begin{array}{l}\text { Ce sont uniquement aux filles que doivent être adressées } \\
\text { les initiatives de prévention de la violence dans les relations } \\
\text { amoureuses (en désaccord) }\end{array}$ & 57 & 98,2 & 277 & 97,8 & 0,039 \\
\hline $\begin{array}{l}\text { Une initiative de prévention pourrait aider les étudiants à } \\
\text { reconnaître des situations de violence (en accord) }\end{array}$ & 57 & 100,0 & 278 & 96,8 & 1,896 \\
\hline $\begin{array}{l}\text { Tous les cégeps devraient offrir des initiatives de prévention de } \\
\text { la violence dans les relations amoureuses des jeunes adultes } \\
\text { (en accord) }\end{array}$ & 57 & 100,0 & 278 & 95,0 & 2,996 \\
\hline $\begin{array}{l}\text { Une initiative de prévention pourrait motiver les étudiants à parler } \\
\text { à quelqu'un s'ils vivent une situation de violence (en accord) }\end{array}$ & 57 & 100,0 & 278 & 93,2 & $4,130^{*}$ \\
\hline $\begin{array}{l}\text { Une initiative de prévention dispensée en milieu collégial pourrait } \\
\text { aider à prévenir la violence dans les relations amoureuses chez } \\
\text { les étudiants (en accord) }\end{array}$ & 57 & 100,0 & 277 & 88,8 & $7,032^{* *}$ \\
\hline $\begin{array}{l}\text { Les étudiants pourraient développer une vision négative des } \\
\text { relations amoureuses suite à une initiative de prévention de la } \\
\text { violence (en désaccord) }\end{array}$ & 57 & 98,2 & 278 & 86,0 & $6,778^{* *}$ \\
\hline $\begin{array}{l}\text { Les initiatives de prévention intéressent fortement les étudiants } \\
\text { (en accord) }\end{array}$ & 56 & 69,6 & 274 & 81,4 & $3,920^{*}$ \\
\hline $\begin{array}{l}\text { Le personnel enseignant et professionnel du cégep est sujet à } \\
\text { recevoir des confidences d'étudiants quant à une situation d'abus } \\
\text { ou de violence (en accord) }\end{array}$ & 57 & 98,2 & 277 & 74,4 & $15,938^{* * *}$ \\
\hline $\begin{array}{l}\text { Les étudiants pourraient être plus réticents à développer une } \\
\text { relation intime avec un partenaire s'ils participent à une initiative } \\
\text { de prévention sur la violence dans les relations amoureuses } \\
\text { (en désaccord) }\end{array}$ & 57 & 78,9 & 273 & 74,4 & 0,532 \\
\hline $\begin{array}{l}\text { Une initiative de prévention soutenue par le personnel enseignant } \\
\text { peut être aussi efficace que si elle est offerte par un ou une } \\
\text { spécialiste dans le domaine (en accord) }\end{array}$ & 55 & 85,5 & 278 & 67,6 & $7,027^{* *}$ \\
\hline $\begin{array}{l}\text { Mes parents vont me comprendre si je vis une situation } \\
\text { amoureuse difficile et que je leur en parle (en accord) }\end{array}$ & --- & --- & 276 & 77,2 & --- \\
\hline $\begin{array}{l}\text { Mes amis sont bien placés pour me dire si ma relation amoureuse } \\
\text { est inégalitaire (en désaccord) }\end{array}$ & --- & --- & 273 & 64,5 & --- \\
\hline $\begin{array}{l}\text { Les parents sont sujets à recevoir des confidences quant à une } \\
\text { situation d'abus et de violence (en accord) }\end{array}$ & --- & --- & 276 & 55,4 & --- \\
\hline
\end{tabular}

$* \leq 0,05 ; * * \leq 0,01 ; * * * \leq 0,001$ 
amoureuses. Alors que la presque totalité des membres du personnel scolaire se considère susceptible de recevoir des confidences de la part des étudiantes et étudiants quant à une situation de violence vécue, près de $75 \%$ des jeunes eux-mêmes partagent ce point de vue. Finalement, 85,5\% des membres du personnel scolaire croient qu'une initiative de prévention soutenue peut être aussi efficace si elle est offerte par le personnel enseignant que si elle est offerte par un ou une spécialiste dans le domaine, alors qu'une proportion moindre (67,6\%) des étudiantes et étudiants sont du même avis. Rappelons que trois énoncés ont été ajoutés au questionnaire s'adressant aux jeunes afin de recueillir leurs perceptions quant au rôle des parents et des pairs. Les données indiquent qu'un peu plus de la moitié des jeunes affirment que les parents sont sujets à recevoir des confidences relativement à une situation de violence vécue et plus des trois quarts considèrent que leurs parents pourraient les comprendre s'ils vivent une situation amoureuse difficile.

\section{Barrières des membres du personnel à aborder la VRA}

Les membres du personnel identifient certains obstacles à la mise en place d'initiatives de prévention. Ces résultats sont présentés au tableau 3. Bien que la vaste majorité considère que le sujet de la VRA est une thématique importante et qu'elles ou qu'ils sont réceptifs à aborder le sujet ou à recevoir les confidences d'une victime, la barrière identifiée par le plus grand nombre de répondantes et répondantes a trait au manque d'outils pour soutenir les initiatives de prévention. De plus, le manque de temps lié à une tâche déjà surchargée est identifié par plus du tiers des répondantes et répondants. Près de la moitié des membres du personnel scolaire indiquent qu'ils aborderaient le thème seulement s'ils croient que la ou le jeune est victime de violence. Plusieurs répondantes et répondants endossent les énoncés témoignant d'un manque de connaissances nécessaires pour soutenir les initiatives de prévention ou pour répondre aux préoccupations des jeunes face à leurs relations amoureuses ou d'un malaise à parler de sexualité avec les jeunes.

Parmi les faits saillants à souligner, notons que près du tiers des répondantes et répondants n'aborderaient pas la question de la VRA à moins que les jeunes initient le sujet et que près du quart craignent qu'une initiative de prévention ne suscite des questions auxquelles elles ou ils ne peuvent répondre. Par ailleurs, un membre sur cinq considère que de répondre aux préoccupations des jeunes quant à la VRA ne fait pas partie de leur rôle ou encore que leur manque d'expérience pour soutenir les initiatives de prévention risque de causer plus de tort que de bien. Finalement la majorité des répondantes et répondants considèrent qu'une initiative de prévention faisant appel aux pairs est une option à privilégier en milieu collégial.

\section{Intérêts des étudiantes et étudiants par rapport aux moyens de promotion des RAHE}

Tel que présenté au tableau 4, les étudiantes et étudiants montrent un intérêt certain à l'égard de l'ensemble des moyens de promotion des RAHE proposés. L'analyse des données révèle que l'intérêt est plus manifeste envers la possibilité de consulter une intervenante ou intervenant de milieu qui pourrait les écouter et les conseiller, entre autres, pour ce qui est des questions relatives aux relations amoureuses. Les étudiantes et étudiants se montrent aussi intéressés à assister à des activités en classe ou hors cours incluant des témoignages sur le thème des relations amoureuses, ou à des activités organisées par des spécialistes dans le domaine. Par contre, les étudiantes et étudiants apparaissent moins nombreux à privilégier le jeu éducatif, les groupes de discussion et les activités d'improvisation comme modalités de promotion des RAHE. 
Tableau 3

Barrières à aborder la violence dans les relations amoureuses selon les membres du personnel

\begin{tabular}{|c|c|c|}
\hline & $N$ & $\%$ \\
\hline $\begin{array}{l}\text { Le personnel enseignant et professionnel manque d'outils pour soutenir des initiatives } \\
\text { de prévention de la violence dans les relations amoureuses (en accord) }\end{array}$ & 55 & 78,2 \\
\hline $\begin{array}{l}\text { J'aborderais le sujet seulement si j'avais des doutes qu'un étudiant soit victime de } \\
\text { violence dans sa relation amoureuse (en désaccord) }\end{array}$ & 57 & 49,1 \\
\hline $\begin{array}{l}\text { J'ai les connaissances nécessaires pour soutenir une initiative de prévention de la } \\
\text { violence dans les relations amoureuses auprès des étudiants (en désaccord) }\end{array}$ & 57 & 47,4 \\
\hline $\begin{array}{l}\text { La tâche du personnel enseignant et professionnel est déjà surchargée. Ces gens } \\
\text { n'ont pas de temps à consacrer pour aborder la question de la violence dans relations } \\
\text { amoureuses (en accord) }\end{array}$ & 55 & 38,2 \\
\hline $\begin{array}{l}\text { Je ne me sens pas compétent à répondre aux préoccupations des étudiants face à leurs } \\
\text { relations amoureuses (en accord) }\end{array}$ & 57 & 35,1 \\
\hline $\begin{array}{l}\text { Si les étudiants n'abordent pas le sujet, je n'initierai pas la question de la violence } \\
\text { dans les relations amoureuses (en accord) }\end{array}$ & 55 & 32,7 \\
\hline $\begin{array}{l}\text { Je ne me sens pas à l'aise à l'idée de parler de relations amoureuses et de sexualité } \\
\text { avec les étudiants (en accord) }\end{array}$ & 57 & 29,8 \\
\hline $\begin{array}{l}\text { Je crains qu'une initiative de prévention sur la violence dans les relations amoureuses, } \\
\text { intégrée en milieu collégial, suscite chez les étudiants des questions auxquelles je ne } \\
\text { saurais pas comment répondre (en accord) }\end{array}$ & 57 & 26,3 \\
\hline $\begin{array}{l}\text { Je considère que ce n'est pas mon rôle de répondre aux préoccupations des étudiants } \\
\text { sur le thème de la violence dans les relations amoureuses (en accord) }\end{array}$ & 57 & 22,8 \\
\hline $\begin{array}{l}\text { Je crains que mon manque d'expérience pour soutenir des initiatives de prévention } \\
\text { de la violence dans les relations amoureuses amène plus de tort que de bien chez les } \\
\text { étudiants (en accord) }\end{array}$ & 57 & 19,3 \\
\hline $\begin{array}{l}\text { Trop peu d'étudiants vivent des situations de violence dans les relations amoureuses } \\
\text { pour en faire un thème de discussion (en accord) }\end{array}$ & 57 & 17,5 \\
\hline $\begin{array}{l}\text { Le fait qu'un étudiant puisse venir me dévoiler une situation de violence me } \\
\text { décourage à aborder le sujet ouvertement (en accord) }\end{array}$ & 57 & 14,0 \\
\hline $\begin{array}{l}\text { Je considère une initiative de prévention faisant appel aux pairs comme étant une } \\
\text { option à privilégier en milieu collégial (en désaccord) }\end{array}$ & 57 & 14,0 \\
\hline
\end{tabular}




\section{Tableau 4}

\section{Intérêts des étudiants et étudiantes par rapport aux moyens de promotion de relations} amoureuses harmonieuses et égalitaires

\begin{tabular}{|c|c|c|c|}
\hline & $N$ & Non $(\%)$ & Oui $(\%)$ \\
\hline \multicolumn{4}{|l|}{ Information } \\
\hline $\begin{array}{l}\text { Capsules sur les relations amoureuses harmonieuses et égalitaires et } \\
\text { dans le Médiavic }\end{array}$ & 278 & 20,9 & 79,1 \\
\hline Brochures distribuées aux étudiants sur les relations amoureuses saines & 278 & 26,3 & 73,7 \\
\hline Proposition de sites Internet sur les relations amoureuses saines & 278 & 26,6 & 73,4 \\
\hline $\begin{array}{l}\text { Kiosques d'informations sur les relations amoureuses harmonieuses et } \\
\text { égalitaires }\end{array}$ & 278 & 37,1 & 62,9 \\
\hline \multicolumn{4}{|l|}{ Animation hors cours ou activité en classe } \\
\hline Témoignages sur le thème des relations amoureuses & 276 & 12,0 & 88,0 \\
\hline $\begin{array}{l}\text { Activités sur les relations amoureuses saines organisées par des } \\
\text { spécialistes (sexologue, psychologue, travailleur social, etc.) }\end{array}$ & 277 & 13,4 & 86,6 \\
\hline Projection d'un film sur le thème des relations amoureuses saines & 276 & 23,9 & 76,1 \\
\hline Proposition de sites Internet sur les relations amoureuses saines & 278 & 28,1 & 71,9 \\
\hline $\begin{array}{l}\text { Présentation d'une pièce de théâtre sur le thème des relations } \\
\text { amoureuses saines }\end{array}$ & 278 & 29,0 & 71,0 \\
\hline $\begin{array}{l}\text { Activités sur les relations amoureuses saines organisées par des } \\
\text { étudiants du cégep }\end{array}$ & 275 & 40,0 & 60,0 \\
\hline Activités sur les relations amoureuses saines réalisées dans les cours & 278 & 46,0 & 54,0 \\
\hline Activités d'improvisation sur le thème des relations amoureuses saines & 277 & 50,5 & 49,5 \\
\hline $\begin{array}{l}\text { Participer à des groupes de discussions sur les relations amoureuses } \\
\text { saines }\end{array}$ & 275 & 52,0 & 48,0 \\
\hline $\begin{array}{l}\text { Participer à un jeu éducatif sur les relations amoureuses harmonieuses et } \\
\text { égalitaires }\end{array}$ & 275 & 56,7 & 43,3 \\
\hline \multicolumn{4}{|l|}{ Consultation } \\
\hline $\begin{array}{l}\text { Présence d'un intervenant de milieu (quelqu'un qui est disponible pour } \\
\text { les étudiants) qui peut nous écouter ou nous conseiller, entre autre quant } \\
\text { aux questions touchant aux relations amoureuses }\end{array}$ & 276 & 3,3 & 96,7 \\
\hline
\end{tabular}




\section{Besoins d'information et d'intervention des étudiantes et étudiants en lien avec la thématique des RAHE}

Les besoins des étudiantes et étudiants en lien avec la thématique des relations amoureuses harmonieuses et égalitaires sont illustrés au tableau 5. Les réponses obtenues révèlent des besoins liés tant à l'information

qu'à l'intervention. À ce propos, les étudiantes et étudiants se montrent particulièrement intéressés aux informations qui pourraient leur être utiles avec leurs pairs. Ainsi, une majorité d'étudiantes et d'étudiants identifient le besoin de connaître les démarches à entreprendre si une personne de leur entourage est victime de violence. Dans cette même optique, ils sont nombreux à se montrer intéressés à distinguer les signes de VRA. Ils sont toutefois moins nombreux à identifier le besoin d'échanger autour des paramètres d'une relation amoureuse saine.

Les résultats relatifs aux besoins d'intervention montrent qu'une proportion importante d'étudiantes et d'étudiants souhaitent questionner leur fonctionnement au sein d'une relation amoureuse. Ainsi, les étudiantes et étudiants mentionnent être intéressés aux interventions qui leur permettraient de préciser leurs limites, de définir leurs besoins et d'améliorer leurs stratégies de communication au sein d'une relation amoureuse. Par ailleurs, une majorité mentionne un intérêt à échanger autour des thèmes de la jalousie et de l'infidélité dans une relation amoureuse.

\section{Intérêts des membres du personnel à l'égard de la prévention de la VRA}

Le tableau 6 fait état des intérêts des membres du personnel à l'égard de la prévention de la VRA. Ainsi, chaque thème proposé a intéressé plus de $90 \%$ de l'échantillon, à savoir l'ampleur et les facteurs de risque associés à la VRA; les indicateurs de situations de VRA et la façon de réagir au dévoilement d'un étudiant ou une étudiante en regard d'une situation de violence. La très grande majorité des membres du personnel se sont montrés intéressés à recevoir une formation en matière de prévention de la VRA. En outre, ils indiquent être intéressés à connaître l'impact de la violence, les différentes ressources disponibles pour les victimes et la façon de soutenir une étudiante ou un étudiant victime de VRA.

\section{DISCUSSION}

Compte tenu de la rareté des initiatives de prévention de la VRA et de promotion des RAHE auprès des jeunes adultes en milieu collégial, cette étude visait à obtenir un regard croisé des perceptions respectives des acteurs impliqués afin de planifier de tels programmes et soulever les enjeux liés à leur implantation. À notre connaissance, bien que de telles études aient été menées auprès de différents acteurs dans le domaine de l'agression sexuelle et de l'éducation à la sexualité, entre autres auprès des infirmières en milieu scolaire de niveau primaire (Hamelin-Brabant et al., 2007), des mères d'enfants d'âge préscolaire (Laforest, 2001) et des enseignantes et enseignants du primaire (St-Vincent, 2002), aucune n'a exploré les perceptions en milieu collégial en regard de la VRA. La présente étude offrait, par ailleurs, la possibilité de contraster les connaissances, les attitudes, les intérêts et les besoins des jeunes et des membres du personnel. 


\section{Besoins d'information et d'intervention des étudiants et étudiantes en lien avec la thématique des relations amoureuses harmonieuses et égalitaires}

\begin{tabular}{|c|c|c|c|}
\hline & $N$ & $\begin{array}{l}\text { Pas ou peu } \\
\text { intéressé }(\%)\end{array}$ & $\begin{array}{l}\text { Intéressé ou très } \\
\text { intéressé }(\%)\end{array}$ \\
\hline \multicolumn{4}{|l|}{ Information } \\
\hline $\begin{array}{l}\text { Savoir quoi faire si quelqu'un de mon entourage vit une situation } \\
\text { de violence dans une relation amoureuse }\end{array}$ & 275 & 6,2 & 93,8 \\
\hline Connaître les signes de violence dans une relation amoureuse & 274 & 9,1 & 90,9 \\
\hline $\begin{array}{l}\text { Distinguer les besoins dans une relation amoureuse harmonieuse } \\
\text { et égalitaire selon le sexe (gars ou fille) }\end{array}$ & 275 & 16,0 & 84,0 \\
\hline Connaître l'impact de la violence dans une relation amoureuse & 276 & 16,3 & 83,7 \\
\hline $\begin{array}{l}\text { Faire l'inventaire des ressources disponibles pour les personnes } \\
\text { qui vivent une situation de violence dans une relation amoureuse }\end{array}$ & 275 & 23,3 & 76,7 \\
\hline Échanger autour des paramètres d'une relation amoureuse saine & 276 & 43,5 & 56,5 \\
\hline \multicolumn{4}{|l|}{ Intervention } \\
\hline Préciser mes limites dans une relation amoureuse & 276 & 14,1 & 85,9 \\
\hline $\begin{array}{l}\text { Échanger autour des thèmes de la jalousie et de l'infidélité dans } \\
\text { une relation amoureuse }\end{array}$ & 276 & 15,2 & 84,8 \\
\hline $\begin{array}{l}\text { Améliorer mes stratégies de communication dans une relation } \\
\text { amoureuse }\end{array}$ & 274 & 15,3 & 84,7 \\
\hline Définir mes besoins dans une relation amoureuse & 276 & 16,3 & 83,7 \\
\hline Réfléchir sur mes valeurs dans une relation amoureuse & 275 & 22,5 & 77,5 \\
\hline Échanger sur la question de l'intimité avec un ou une partenaire & 275 & 27,6 & 72,4 \\
\hline $\begin{array}{l}\text { Réfléchir sur les avantages d'une relation amoureuse } \\
\text { harmonieuse et égalitaire }\end{array}$ & 276 & 28,3 & 71,7 \\
\hline Parler de l'amour & 275 & 28,4 & 71,6 \\
\hline $\begin{array}{l}\text { Échanger autour des caractéristiques d'une relation amoureuse } \\
\text { égalitaire et harmonieuse selon le sexe (gars ou fille) }\end{array}$ & 276 & 33,3 & 66,7 \\
\hline Échanger autour de l'expérience de l'intimité affective & 276 & 34,1 & 65,9 \\
\hline $\begin{array}{l}\text { Échanger autour des caractéristiques d'une relation amoureuse } \\
\text { harmonieuse et égalitaire selon les orientations sexuelles }\end{array}$ & 275 & 36,4 & 63,6 \\
\hline $\begin{array}{l}\text { Réfléchir sur l'influence des valeurs culturelles sur une relation } \\
\text { amoureuse }\end{array}$ & 276 & 37,0 & 63,0 \\
\hline
\end{tabular}




\section{Tableau 6}

Intérêts des membres du personnel à l'égard de la prévention de la violence dans les relations amoureuses

\begin{tabular}{lccc}
\hline & $N$ & $\begin{array}{c}\text { Pas ou peu } \\
\text { intéressé (\%) }\end{array}$ & $\begin{array}{c}\text { Intéressé ou très } \\
\text { intéressé (\%) }\end{array}$ \\
\hline $\begin{array}{l}\text { Ampleur et facteurs de risque associés à la violence dans les } \\
\text { relations amoureuses }\end{array}$ & 57 & 0,0 & 100,0 \\
$\begin{array}{l}\text { Les indicateurs de situations de violence dans les relations } \\
\text { amoureuses }\end{array}$ & 57 & 1,8 & 98,2 \\
$\begin{array}{l}\text { Comment réagir au dévoilement d'un étudiant en regard d'une } \\
\text { situation de violence }\end{array}$ & 57 & 3,5 & 96,5 \\
$\begin{array}{l}\text { Impact de la violence dans les relations amoureuses chez les } \\
\text { étudiants }\end{array}$ & 57 & 5,3 & 94,7 \\
$\begin{array}{l}\text { Les ressources disponibles pour les victimes } \\
\begin{array}{l}\text { Comment soutenir un étudiant victime de violence dans sa relation } \\
\text { amoureuse }\end{array}\end{array}$ & 57 & 7,0 & 93,0 \\
\hline $\begin{array}{l}\text { Dans l'éventualité où une telle formation serait offerte par votre } \\
\text { cégep est-ce que vous seriez intéressés à y participer? (Non/Oui) }\end{array}$ & 57 & 3,5 & 93,0 \\
\hline
\end{tabular}

Les données recueilles permettent de constater que les deux groupes ont, en général, des connaissances justes par rapport au phénomène de la VRA. Ils savent, par exemple, que la violence peut être présente malgré l'absence d'actes témoignant de violence physique; qu'il est faux de penser que la victime a provoqué la situation de violence; que les gestes violents tendent à s'intensifier avec le temps; que la victime craint de dévoiler les actes et qu'il est difficile pour elle de mettre un terme à la relation. Près de $15 \%$ considèrent, à tort, que les victimes dévoilent peu de temps après l'événement et que les conséquences associées à la VRA ne sont pas aussi sévères que celles associées à la violence conjugale. Dans les faits, les victimes de violence sont peu nombreuses à dévoiler leur situation, en particulier à des figures adultes, en raison du stigma social et culturel associé à la VRA (Cornelius et Resseguie, 2007). Plusieurs conséquences négatives sont associées à la VRA chez les jeunes. D'une part, la VRA est associée à de nombreux problèmes d'adaptation, et d'autre part, une première expérience de violence peut accroître les risques de revictimisation dans les relations ultérieures (Vézina et Hébert, 2007). Enfin, peu de différences sont identifiées entre les taux de bonnes réponses des étudiantes et étudiants et des membres du personnel scolaire. Par contre, trois éléments de contenu semblent moins bien intégrés et devraient être abordés dans les programmes destinés aux jeunes en milieu collégial, à savoir la question du genre et de la scolarité comme facteur de risque et du fait que la plupart des cas ne sont pas rapportés aux autorités policières.

En ce qui concerne les attitudes à l'égard de la promotion des RAHE, les deux groupes entretiennent généralement des attitudes positives quant aux bénéfices potentiels des initiatives de prévention. Nous pouvons 
donc entrevoir une réception favorable à de telles initiatives en milieu collégial. Par contre, un pourcentage non négligeable considère que les initiatives de prévention peuvent être associées à certains effets négatifs, notamment que les participantes et participants seraient plus réticents à développer une relation intime avec un partenaire. Ces réticences devraient être approfondies lors d'études ultérieures de façon à mieux saisir les enjeux dans la pratique.

Dans un même ordre d'idées, Lavoie (2000) fait valoir l'importance de situer les interventions de prévention dans un cadre plus global d'activités de promotion des relations égalitaires sans violence et de choix respectueux des autres dans les relations interpersonnelles et intimes. Cette avenue aurait l'avantage de présenter aux jeunes des modèles amoureux alternatifs et de situer leurs attentes et leurs besoins face à ceux-ci. Les données révèlent certaines distinctions entre les perceptions des membres du personnel et des étudiantes et étudiants, notamment quant à l'intérêt des jeunes de participer à des initiatives de prévention et la probabilité qu'un membre du personnel soit appelé à recevoir des confidences d'un ou une jeune victime de violence. Ainsi les jeunes eux-mêmes se déclarent comme davantage intéressés aux initiatives de prévention que les membres du personnel estiment qu'ils le sont. Par ailleurs, les membres du personnel se perçoivent comme davantage susceptibles de recevoir les confidences des jeunes que ce que les jeunes rapportent eux-mêmes. Une autre distinction réfère au fait que les jeunes croient que les interventions dispensées par des spécialistes du domaine sont plus efficaces que les interventions données par les membres du personnel enseignant.

Par ailleurs, bien que les membres du personnel considèrent la VRA comme une thématique cruciale, ils identifient des barrières dont les plus importantes semblent être le manque d'outils pour soutenir les initiatives de prévention et le manque de temps lié à une tâche déjà surchargée. À ce propos, Bowen et Desbiens (2004) mentionnent au sujet de la prévention de la violence en milieu scolaire que compte tenu de la tâche des enseignants et enseignantes et d'un roulement important de personnel, maintenir l'intérêt et assurer le suivi à l'égard des besoins de formation constituent de réels défis dans l'implantation des programmes. Il a été démontré que l'implantation des programmes de prévention est accrue lorsque les membres du personnel sont sensibles et compétents et reçoivent une formation adéquate, du soutien et de la supervision (Nation et al., 2003).

Dans un même ordre d'idées, une proportion importante (près du quart) craint qu'une initiative de prévention ne suscite des questions auxquelles ils ne pourraient répondre. Près du tiers des répondantes et répondants ne se sentent pas compétents ou à l'aise pour aborder le thème des relations amoureuses et de la sexualité. Ces résultats font écho à d'autres travaux (Cohen, Byers et Sears, 2012; Ninomiya, 2010) qui font état du malaise des enseignantes et enseignants non spécialistes pour aborder des contenus liés à la santé sexuelle. Une attention particulière doit être portée à ce malaise exprimé et donc s'assurer qu'ils aient la formation nécessaire non seulement au plan du savoir-faire, mais également au plan du savoir-être pour développer plus d'aisance avec ce type de contenu. Une collaboration avec du personnel spécialisé (sexologue, intervenante et intervenant communautaire qui dispense des programmes, etc.) du milieu pourrait être une autre avenue à privilégier.

La majorité des répondantes et répondants (86\%) estiment qu'une initiative faisant appel aux pairs est à privilégier. L'éducation par les pairs est une stratégie reconnue auprès des jeunes du collégial et à considérer en prévention de la violence puisqu'elle aurait un effet significatif sur la norme du groupe de 
pairs (Banyard, Moynihan et Crossman, 2009). Par contre, une méta-analyse des programmes éducatifs en matière d'agression sexuelle (Anderson et Whiston, 2005) semble suggérer que les interventions offertes par des professionnelles et professionnels sont plus efficaces que celles dispensées par des pairs. Certaines hypothèses ont été avancées pour expliquer ce constat, à savoir le manque d'investissement dans l'éducation par les pairs pouvant être considérée comme de la main-d'œuvre à bon marché, le manque de reconnaissance de la complexité du processus d'éducation par les pairs et le besoin de pouvoir compter sur un personnel hautement qualifié qui dispose d'une supervision adéquate, d'un entraînement rigoureux, et d'un soutien suffisant pour assurer un suivi éventuel, ce qui n'est pas toujours le cas.

Soulignons que plusieurs études empiriques soutiennent qu'une majorité de victimes se confient d'abord à leurs pairs lorsqu'ils vivent une situation de violence (Weisz et Black, 2010). Les travaux effectués auprès des étudiantes et des étudiants de niveau collégial tendent à démontrer le rôle clé des pairs aidants au plan tant de la prévention de la victimisation que du soutien aux victimes (Banyard et al., 2009). Les interventions de prévention auraient alors avantage à préparer les pairs à agir comme trait d'union et en complémentarité aux ressources du milieu. Les jeunes seraient alors en mesure de repérer un pair qui vit de la violence, l'informer sur les ressources disponibles, l'encourager et l'accompagner à prendre contact avec les intervenants et intervenantes en place (Fernet, 2005).

En ce qui concerne les intérêts par rapport aux moyens de promotion des RAHE, la présence d'une intervenante ou d'un intervenant de milieu (quelqu'une ou quelqu'un qui est disponible pour les étudiantes et étudiants) qui peut écouter ou conseiller est l'option privilégiée par la vaste majorité des étudiantes et étudiants (97\%). À cet effet, Nation et ses collègues (2003), qui ont mené une recension des programmes de prévention qui se sont avérés efficaces auprès des jeunes dans quatre domaines (abus de substances, comportements sexuels à risque, échecs scolaires et décrochage, délinquance et violence), soulignent l'efficacité des programmes qui misent sur le développement de relations positives avec des figures adultes. Parmi les activités sélectionnées par la grande majorité, on note des activités (en classe/à l'extérieur) qui impliquent des témoignages sur le thème des relations amoureuses ou des activités organisées par des spécialistes (sexologue, psychologue, travailleuse ou travailleur social, etc.).

Relativement aux besoins en lien avec la thématique des relations amoureuses harmonieuses et égalitaires, les étudiantes et étudiants reconnaissent avoir des besoins liés à l'information et l'intervention. Ils semblent afficher un intérêt plus marqué pour des informations sur les démarches à entreprendre si une personne de l'entourage vit une situation de VRA, sur les signes et l'impact de la violence et sur les moyens de distinguer les besoins dans une relation amoureuse selon le sexe. Sur le plan des besoins d'intervention, les étudiantes et étudiants semblent particulièrement intéressés aux initiatives leur permettant de préciser leurs limites dans une relation amoureuse, d'échanger autour des thèmes de la jalousie et de l'infidélité et d'améliorer leurs stratégies de communication. D'ailleurs, des travaux récents réalisés auprès d'adolescents et adolescentes et de jeunes adultes suggèrent que la confiance en l'autre et la jalousie sont les sources de conflits les plus souvent rapportées en contexte amoureux, tant par les garçons que par les filles, et font valoir l'importance de promouvoir les habiletés de communication en situation de conflits dans les programmes de sensibilisation et de prévention de la VRA (Fernet, Hébert, Gascon et Lacelle, 2012).

Finalement, relativement aux intérêts à l'égard de la prévention de la VRA, la très grande majorité du personnel enseignant $(96,5 \%)$ se dit intéressée à participer à une formation. Tous les thèmes proposés 
intéressent la très grande majorité des membres du personnel scolaire. Ces résultats témoignent de l'ouverture du milieu et renforcent la nécessité d'offrir des activités de formation et de développer des outils pédagogiques pour soutenir ces efforts de prévention de la VRA et de promotion des RAHE auprès des jeunes de niveau collégial. Malgré ces défis liés à un horaire déjà très chargé, il semble donc pertinent de poursuivre l'élaboration d'outils visant à soutenir la prévention afin de répondre aux besoins exprimés. Comme d'autres l'ont aussi fait remarquer (Lavoie et al., 2012), il serait utile qu'on puisse retrouver au Québec des programmes de prévention universelle de la VRA, à multiples composantes et offerts de façon continue sur plusieurs années dans l'ensemble des établissements scolaires.

\section{CONCLUSION}

Cette étude a fourni certaines pistes de réflexion quant à l'implantation d'activités de prévention de la VRA et de promotion de RAHE chez les jeunes du collégial tout en identifiant les perceptions communes et distinctes des jeunes eux-mêmes et des intervenants et intervenantes du milieu scolaire. Mentionnons toutefois que la présente étude comporte certaines limites. Elle repose sur un sous-échantillon de convenance qui n'est pas représentatif de la population étudiante collégiale. Les contraintes liées à l'obtention du consentement parental pour les mineurs ont affecté la participation des étudiantes et étudiants plus jeunes en début de cheminement. Pour ce qui est des membres du personnel, une importante proportion de participantes et participants sont des enseignantes et enseignants évoluant dans le programme des techniques humaines et ont été recrutés dans le cadre d'activités de sensibilisation aux RAHE. Ces participantes et participants pourraient présenter d'emblée une attitude favorable envers cette thématique. Ajoutons que peu d'outils de mesure validés sont disponibles pour mesurer les connaissances, les attitudes, les intérêts et les besoins des jeunes de niveau collégial face à la prévention de la VRA et à la promotion des RAHE. Les instruments de mesure destinés aux membres du personnel sont encore plus rares et, à notre connaissance, aucun n'a été développé dans le contexte spécifique de la prévention de la VRA.

Malgré ces limites, cette étude offre des pistes pertinentes pour les promoteurs de programmes qui souhaitent mettre en place des activités de prévention de la VRA destinés aux jeunes de niveau collégial. Par ailleurs, il importe de poursuivre la réflexion amorcée par des recherches ultérieures. Ainsi, les études futures devraient cerner chez les membres du personnel scolaire au collégial le rôle de la formation, de l'expérience, des connaissances et des attitudes associées à l'éducation sexuelle, variables prédisant l'aisance des enseignants et enseignantes du primaire et du secondaire à livrer ce type de contenu (Cohen et al., 2012). Par ailleurs, des groupes de discussion conjoints impliquant tant des participantes et participants étudiants que des membres du personnel pourraient permettre de mieux cerner les barrières respectives de chacun et favoriser le dialogue de manière à stimuler la recherche d'approches concertées. 


\section{RÉFÉRENCES}

Anderson, L. A. et Whiston, S. C. (2005). Sexual assault education programs: A meta-analytic examination of their effectiveness. Psychology of Women Quarterly, 29(4), 374-388. doi: 10.1111/j.1471-6402.2005.00237.x

Avery-Leaf, S., Cascardi, M., O’Leary, K. D. et Cano, A. (1997). Efficacy of a dating violence prevention program on attitudes justifying aggression. Journal of Adolescent Health, 21(1), 11-17. doi: 10.1016/S1054-139X(96)00309-6

Banyard, V. L., Moynihan, M. M. et Crossman, M. T. (2009). Reducing sexual violence on campus: The role of student leaders as empowered bystanders. Journal of College Student Development, 50(4), 446-457. doi: 10.1353/ csd.0.0083

Banyard, V. L., Plante, E. G. et Moynihan, M. M. (2004). Bystander education: Bringing a broader community perspective to sexual violence prevention. Journal of Community Psychology, 32(1), 61-79. doi: 10.1002/jcop.10078

Bowen, F. et Desbiens, N. (2004). La prévention de la violence en milieu scolaire au Québec : réflexions sur la recherche et le développement de pratiques efficaces. Éducation et Francophonie, 32(1), 69-86. Consulté à http://www. acelf.ca/c/revue/pdf/LapreventionQuebec.pdf

Centre national d'information sur la violence dans la famille du Canada. (2006). La violence dans les fréquentations : aperçu. Ottawa, ON : Agence de santé publique du Canada. Consulté à http://www.phac-aspc.gc.ca/ncfv-cnivf/ sources/fem/fem-relations/index-fra.php

Cohen, J. N., Byers, S. E. et Sears, H. A. (2012). Factors affecting Canadian teachers' willingness to teach sexual health education. Sex Education, 12(3), 299-316. doi: 10.1080/14681811.2011.615606

Cornelius, T. L. et Resseguie, N. (2007). Primary and secondary prevention programs for dating violence: A review of literature. Aggression and Violent Behavior, 12(3), 364-375. doi: 10.1016/j.avb.2006.09.006

Fernet, M. (2005). Amour, violence et adolescence. Montréal : Presses de l'Université du Québec.

Fernet, M., Hébert, M., Gascon, S. et Lacelle, C. (2012). Agressions sexuelles et comportements sexuels à risque. Dans M. Hébert, M. Cyr et M. Tourigny (Éds.), Les agressions sexuelles envers les enfants (Tome II, p. 131-170). Sainte-Foy: Presses de l'Université du Québec.

Hamelin-Brabant, L., Damant, D., Michaud, F. et Alain, M.-C. (2007). Analyse des besoins de formation des infirmières en milieu scolaire concernant la prévention des agressions sexuelles auprès des élèves de niveau scolaire primaire. Montréal, QC : CRI-VIFF, Collection études et analyses.

Krajewski, S. S., Rybarik, M. F., Dosch, M. F. et Gilmore, G. D. (1996). Results of a curriculum intervention with seventh graders regarding violence in relationships. Journal of Family Violence, 11(2), 93-112. doi : 10.1007/BF02336664

Laforest, M. S. (2001). Étude exploratoire des besoins de formation des mères d'enfants d'âge préscolaire en matière de prévention de l'abus sexuel. Rapport d'activité présenté comme exigence partielle de la maitrise en sexologie, Université du Québec à Montréal, Montréal, QC.

Lavoie, F. (2000). La prévention de la violence dans les relations de couple à l'adolescence. Dans F. Vitaro et C. Gagnon (dir.), Prévention des problèmes d'adaptation chez les enfants et les adolescents : Tome II. Les problèmes externalisés (p. 405-450). Sainte-Foy : Presses de l’Université du Québec.

Lavoie, F., Hébert, M. et Beaulieu-Denault, O. (2012, mars). Pour des relations amoureuses harmonieuses à l'adolescence : un bilan des approches de prévention. Les cahiers de $P V$ : antennes sur la victimologie, $\mathrm{n}^{\circ} 8,40-47$.

Lavoie, F., Hotton-Paquet, V., Laprise, S. et Joyal Lacerte, F. (2009). ViRAJ : Programme de prévention de la violence dans les relations amoureuses chez les jeunes et de promotion des relations égalitaires ( $2^{\mathrm{e}}$ éd.). Québec, QC : Université Laval.

Lavoie, F., Pacaud, M.-C., Roy, M. et Lebossé, C. (2007). PASSAJ : Programme de prévention et de promotion traitant de la violence dans les relations amoureuses et du harcèlement sexuel auprès des jeunes de 16-17 ans. ( $2^{\mathrm{e}}$ éd.) Québec, QC : Université Laval.

Lavoie, F. et Vézina, L. (2002). Violence dans les relations amoureuses à l'adolescence. Dans J. Aubin, C. Lavallée, J. Camirand, N. Audet, B. Beauvais et P. Berthiaume (dir.), Enquête sociale et de santé auprès des enfants et des adolescents québécois 1999 (p. 471-484). Québec : Institut de la statistique du Québec.

Lavoie, F., Vézina, L., Gosselin, A. et Robitaille, L. (1994). ViRAJ: Programme de prévention de la violence dans les relations amoureuses des jeunes. Québec: Gouvernement du Québec, Ministère de l'Éducation.

Ministère de la Sécurité publique du Québec. (2009). La criminalité dans un contexte conjugal-statistiques 2008. Consulté à http://www.inspq.qc.ca/violenceconjugale/statistiques/default.asp?id=6 
Nation, M., Crusto, C., Wandersman, A., Kumpfer, K. L., Seybolt, D. et Morissey-Kane, E. (2003). What works in prevention: Principles of effective prevention programs. American Psychologist, 28(6/7), 449-456. doi: 10.1037/0003-066X.58.6-7.449

Ninomiya, M. M. (2010). Sexual health education in Newfoundland and Labrador schools: Junior high school teachers' experiences, coverage of topics, comfort levels and views about professional practice. Canadian Journal of Human Sexuality, 19(1/2), 15-26.

Riberdy, H. et Tourigny, M. (2009). Violence et fréquentations amoureuses au secondaire : coup d'œil à Montréal : enquête sur le bien-être des jeunes Montréalais. Rapport thématique $\mathrm{n}^{\circ} 3$. Montréal, QC : Direction de santé publique.

St-Vincent, G. (2002). Analyse de besoins de formation des enseignants et enseignantes du primaire en prévention des abus sexuels à l'égard des enfants. Rapport d'activité présenté comme exigence partielle de la maîtrise en sexologie, Université du Québec à Montréal, Montréal, QC.

Ting, S-M. R. (2009). Meta-analysis on dating violence prevention among middle and high schools. Journal of School Violence, 8(4), 328-337. doi: 10.1080/15388220903130197

Vézina, J. et Hébert, M. (2007). Risk factors for victimization in romantic relationships of young women: A review of empirical studies and implications for prevention. Trauma, Violence \& Abuse, 8(1), 33-66. doi: $10.1177 / 1524838006297029$

Weisz, A. N. et Black, B. M. (2010). Peer education and leadership in dating violence prevention: Strengths and challenges. Journal of Aggression, Maltreatment \& Trauma, 19(6), 641-660. doi :10.1080/10926771.2010.502089 